\title{
Increasing Clozapine Dispensing Trends in Queensland, Australia 2004-2013
}

Authors

Affiliations

\author{
T. Forrester ${ }^{1,4}$, D. Siskind ${ }^{2,3}$, K. Winckel ${ }^{1,4}$, A. Wheeler ${ }^{5,6}$, S. Hollingworth
}

Affiliation addresses are listed at the end of the article

\author{
Key words \\ - clozapine \\ - antipsychotic \\ - pharmacoepidemiology \\ - schizophrenia
}

$\begin{array}{ll}\text { received } & 22.03 .2015 \\ \text { revised } & 18.05 .2015 \\ \text { accepted } & 19.05 .2015\end{array}$

Bibliography

DOI http://dx.doi.org/

10.1055/s-0035-1554713

Published online:

June 19, 2015

Pharmacopsychiatry 2015; 48: 164-169

(c) Georg Thieme Verlag KG Stuttgart · New York ISSN 0176-3679

Correspondence

A/Prof. D. Siskind

Mobile Intensive Rehabilitation

Team

519 Kessels Rd

MacGregor

Qld, 4109

Australia

d.siskind@uq.edu.au

\section{Abstract}

$\nabla$

Introduction: Clozapine is the most effective treatment for treatment-resistant schizophrenia but its use is suboptimal.

Methods: Clozapine dispensing data from Queensland, Australia were extracted (20042013). The number of people dispensed clozapine each year and mean maintenance doses were calculated. The 18-week and 5-year cessation and treatment interruption rates were calculated using Kaplan-Meier analysis.

Results: Clozapine dispensings increased 36.4\% $(\mathrm{p}<0.001)$ from 44 to 60 people per 100000 . This was estimated as $8.3 \%$ of people with schizophre-

\section{Introduction}

$\nabla$

Clozapine is the most effective treatment for treatment resistant schizophrenia (TRS) $[1,2]$. It reduces mortality, hospitalisation rates and the risk of suicide [1]. Clozapine's use, however, has been limited by life-threatening adverse drug reactions (ADRs) [1]. These include haematological ADRs such as agranulocytosis and neutropenia, which has resulted in strict monitoring of full blood counts for the duration of treatment [1]. Worldwide estimates of clozapine use vary from $0.04 \%$ in parts of Canada to $60 \%$ in China $[3,4]$. It is expected that $50-60 \%$ of people with TRS will respond to clozapine [5]. Given the efficacy of clozapine for TRS, it has been suggested that clozapine is underused [1].

\section{Objective \\ $\nabla$}

This paper explores trends in clozapine use by analysing de-identified dispensing data extracts for all people dispensed clozapine from public hospitals in Queensland, Australia between 2004 nia and $33.3 \%$ of people with treatment resistant schizophrenia dispensed clozapine in 2013. Mean maintenance dose did not significantly change (364-399 mg) over 5 years of treatment. One in 7 (14.2\%) people ceased within the first 3 weeks. 3-quarters $(72.7 \%)$ reached maintenance therapy. The 5-year actuarial estimate of the proportion of people a) dispensed clozapine was 0.610 (S.E. 0.011) and b) with an interruption to treatment was 0.422 (S.E. 0.013).

Discussion: The number of patients being dispensed clozapine increased between 2004 and 2013 but clozapine is still underused. Increased use combined with continued monitoring for adverse effects will improve quality use of clozapine.

and 2013. It estimates the proportion of people with TRS being dispensed clozapine, and calculates the mean maintenance dose, changes in dosing for individuals over time, and the duration of treatment and interruptions to treatment.

\section{Materials and Methods \\ $\nabla$}

\section{Dataset development}

A dispensing was defined as the supply of a defined quantity of one strength of clozapine to an individual. A dispensing occasion was defined as the total amount of clozapine supplied to an individual on a single occasion. Individual level dose per dispensing occasion was calculated. Only outpatient and discharge dispensings were considered for calculation of doses and duration, as inpatient supplies were irrespective of the patient's dose. An exception was made for longstay mental health hospitals where inpatient dispensings were included in the analysis as they provided a one-month supply at a time calculated by the patient's current dose. 
During the study period, clozapine could only be dispensed in Queensland by a pharmacy associated with a hospital. In Queensland public hospitals, all clozapine dispensings have been recorded in iPharmacy ${ }^{\circledR}$ (the statewide dispensing software) since 2003. We extracted retrospective, de-identified data for all people dispensed clozapine in Queensland over a 10-year period (1 January 2004 to 31 December 2013). The extracted data included date of dispensing, formulation strength, quantity and dispensing type (inpatient vs. outpatient).

In Australia, clozapine is available as a Section 100 Highly Specialised Drug on the Pharmaceutical Benefits Scheme (PBS), consisting of PBS public dispensings (from a public hospital pharmacy) and PBS private dispensings (from private hospitals and community pharmacies associated with hospitals) [6]. Although aggregated data on all PBS clozapine dispensings in Australia are publicly available, they do not show the dispensing date and quantity, and consequently the total amount of clozapine supplied to an individual on a single dispensing occasion cannot be calculated from these data. In 2010, clozapine dispensing began to shift from Queensland public hospitals to Queensland private hospitals and community pharmacies associated with a hospital. Consequently, we needed to include data from both public hospital and non-public hospital sources to calculate the number of people accessing clozapine.

\section{(i) Number of people dispensed clozapine-iPharmacy ${ }^{\circledR}$} The details of clozapine dispensings were extracted from iPharmacy ${ }^{\circledR}$ for all 50 Queensland public hospitals that dispensed clozapine. The absolute number of people dispensed clozapine in the study period was identified. Individuals with dispensings prior to 1 May 2004 were assumed to have been initiated on clozapine before the start of the study period and were excluded from analyses of individuals newly commencing clozapine. Rates of use per 100000 population were calculated using Queensland population data by year [7]. The estimated lifetime prevalence of schizophrenia (7.2 per 1000 persons [8]) and prevalence of TRS (25-33\% [9]) were used to calculate the proportion of people with TRS in Queensland being dispensed clozapine from a public hospital pharmacy. We used linear regression to identify trends in the number of people dispensed clozapine from 2004 to 2013. The residuals were analysed to test the assumptions that they were normally distributed and homoscedastic. Robust standard error was used as auto-correlation may be present in the data and this can bias the standard errors. A p-value $<0.05$ was considered statistically significant.

\section{(ii) Number of people dispensed clozapine - non- iPharmacy ${ }^{\circledR}$}

The data on all PBS dispensings from private hospitals and community pharmacies associated with a hospital in Queensland (hereafter referred to as non- iPharmacy ${ }^{\circledR}$ supply) were examined for the study period [10]. Individual level data were not available for non-iPharmacy ${ }^{\circledR}$ data. Using iPharmacy ${ }^{\circledR}$ and noniPharmacy ${ }^{\circledR}$ clozapine supply, the proportion of clozapine dispensed in the state as recorded by iPharmacy ${ }^{\circledR}$ was calculated. We used linear regression to identify trends in the number of non-iPharmacy ${ }^{\circledR}$ prescriptions dispensed. The residuals were analysed to test the assumptions of linear regression. A p-value $<0.05$ was considered statistically significant.

\section{Mean maintenance dose}

The prescription supply frequency and quantities of clozapine are outlined in the Australian registration documentation. Following initiation, an individual receives 18 weeks of a 7-day supply aligned with weekly blood monitoring (defined in this study as initiation treatment); and after this, they progress to a 28-day dispensed supply similarly aligned with weekly blood monitoring for 4 weeks (defined in this study as maintenance treatment). The quantity and strength of tablets dispensed on each occasion for each person on weekly maintenance treatment for 4 weeks was used to calculate a mean dose per person per year after starting clozapine. From this, a mean maintenance dose was calculated for the clozapine treatment population on a yearly basis. The population mean dose in Year 1 was compared to the mean dose in Year 5 and Year 10, respectively, using paired t-tests.

\section{Duration of clozapine treatment}

A discontinuation date was defined as the last dispensing an individual received in the iPharmacy ${ }^{\circledR}$ dataset. People who received an initial dispensing before 1 May 2004 were excluded from the analysis as we assumed these people were taking clozapine before the study period. They were excluded to ensure analyses only included known initiation dates. The actuarial estimate of the proportion of people still dispensed clozapine at the 18-month, 5-year and 9.5-year time points, and the median duration, were estimated using the standard Kaplan-Meier method.

\section{Interruptions to clozapine treatment}

Interruptions to treatment were calculated for all people reaching maintenance therapy (>18 weeks). We defined 2 types of interruptions as per the clozapine registration directions. A short interruption occurred if a clozapine dose was missed for more than $72 \mathrm{~h}$ but less than 28 days. This triggered a change from 28-day maintenance dispensings to 6 weekly dispensings of 7-days' supply. A long interruption occurred if a clozapine dose was missed for more than 28 days. This triggered a return to 18 weekly dispensings of 7-days' supply. For those people who had both short and long interruptions, the time to first interruption was used. Actuarial estimates of the time to first short or long interruption were estimated using the standard Kaplan-Meier method.

Data analysis was conducted using SPSS ${ }^{\circledR}$ version 22 (SPSS Inc., Chicago, IL, USA). Ethics approval for this study was obtained from the Metro South Health, Human Ethics Research Committee (HREC/13/QPAH/292).

\section{Results \\ $\nabla$ \\ Number of people dispensed clozapine}

The iPharmacy ${ }^{\circledR}$ dataset identified 4813 individuals supplied clozapine from Queensland public hospitals between 1 January 2004 and 31 December 2013. Within this clozapine treatment population, 3484 individuals initiated clozapine treatment during the study period ( $\odot$ Fig. 1).

There was a $36.4 \%$ increase ( 2.5 people per 100000 population per year; 95\% CI 2.1-2.9; $\mathrm{p}<0.001$ ) from 44 to 60 people per 100000 between 2004 and 2011. The assumptions of linear regression were tested and the residuals were normally distrib- 


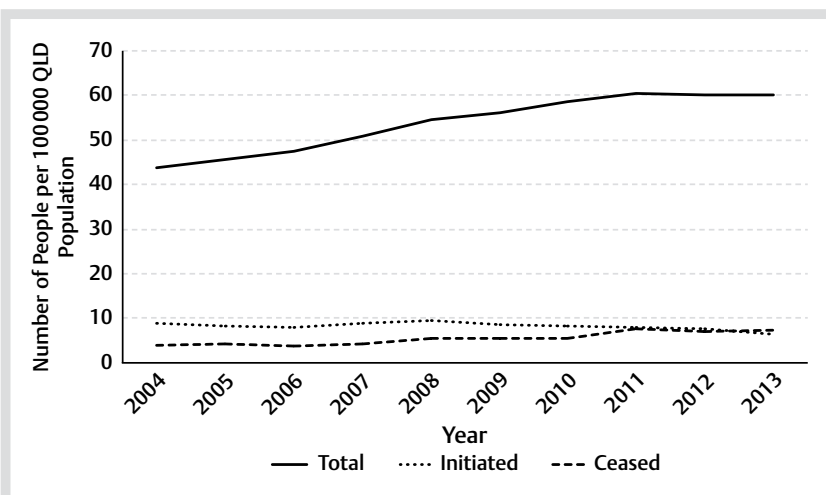

Fig. 1 Number of people per 100000 population dispensed clozapine in Queensland (QLD) between 2004 and 2013.

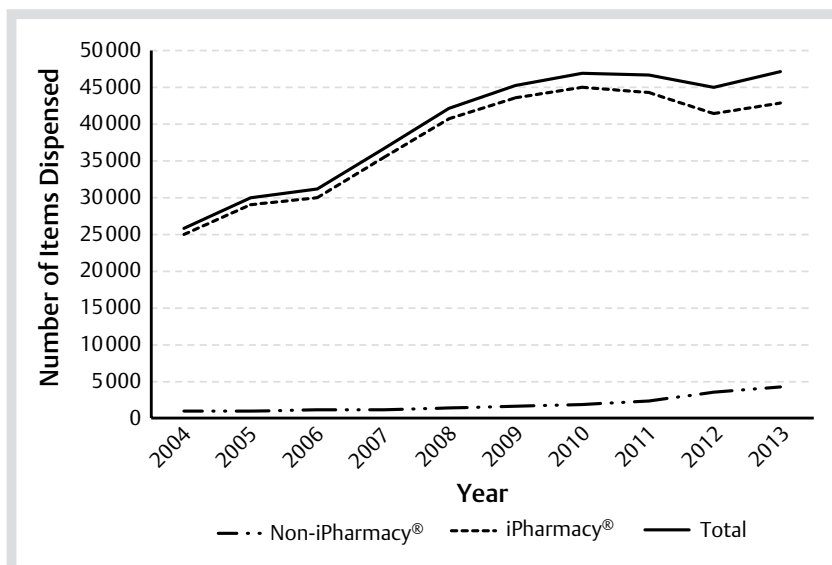

Fig. 2 Number of clozapine items ${ }^{\dagger}$ dispensed in Queensland between 2004 and 2013. ${ }^{\dagger}$ A clozapine item was defined as each individual strength of clozapine dispensed to the person. Note: Non-iPharmacy ${ }^{\circledR}$ dispensings were extracted from PBS data and included dispensing from private hospitals and community pharmacies.

uted and homoscedastic. From 2011, the number of people dispensed clozapine plateaued at 60 per 100000 .

From 2004 to 2011 the number of iPharmacy ${ }^{\circledR}$ dispensings increased by 3130 per year $(95 \%$ CI $2312-3948 ; \mathrm{p}<0.001)$. From 2011 to 2013, there was a decrease in the number of iPharmacy ${ }^{\circledR}$ dispensings but it was not statistically significant.

We also examined non-iPharmacy ${ }^{\circledR}$ dispensing data for the study period. Clozapine non-iPharmacy ${ }^{\circledR}$ prescription dispensings increased by 855 per year from 2010-2013 (95\% CI $586-1124 ; \mathrm{p}<0.005)$. This increase in non-iPharmacy ${ }^{\circledR}$ dispensings corresponded to a decrease in the number of dispensings in the iPharmacy ${ }^{\circledR}$ dataset ( $\odot$ Fig. 2). In 2008, $96.7 \%$ of all clozapine dispensed in Queensland was captured in the iPharmacy ${ }^{\circledR}$ dataset and this decreased to $90.9 \%$ in 2013.

The estimated prevalence of schizophrenia in Queensland was 720 people per 100000 population (using a 7.2 per 1000 persons' lifetime morbid prevalence of schizophrenia) and $8.3 \%$ of these people were dispensed clozapine from Queensland public hospitals. The estimated prevalence of TRS in Queensland was $180-240$ people per 100000 population (25-33\% prevalence of TRS). The proportion of people with TRS dispensed clozapine from Queensland public hospitals increased from 24.4-33.3\% between 2004 and 2013 using the more conservative lower bound TRS prevalence estimate of 180 per 100000 and from
Table 1 Clozapine maintenance dose (mg per day) for each year after a person was first dispensed clozapine.

\begin{tabular}{|ccc|}
\hline $\begin{array}{l}\text { Time on } \\
\text { clozapine (years) }\end{array}$ & $\begin{array}{l}\text { Number of individuals } \\
\text { dispensed clozapine as } \\
\text { maintenance therapy }\end{array}$ & $\begin{array}{l}\text { Mean dose } \\
\mathbf{m g} / \text { day (SD) }\end{array}$ \\
\hline 1 & 2304 & $364(164)$ \\
\hline 2 & 1833 & $379(166)$ \\
\hline 3 & 1445 & $389(170)$ \\
\hline 4 & 1185 & $392(172)$ \\
\hline $5^{\dagger}$ & 962 & $399(171)$ \\
\hline 6 & 741 & $399(173)$ \\
\hline 7 & 554 & $404(173)$ \\
\hline 8 & 397 & $407(186)$ \\
\hline 9 & 247 & $419(185)$ \\
\hline $10^{\ddagger}$ & 95 & $412(156)$ \\
\hline
\end{tabular}

$\dagger$ Increase from Year 1 to Year 5 not statistically significant $(p=0.568)$

$\ddagger$ Increase from Year 1 to Year 10 not statistically significant $(p=0.452)$

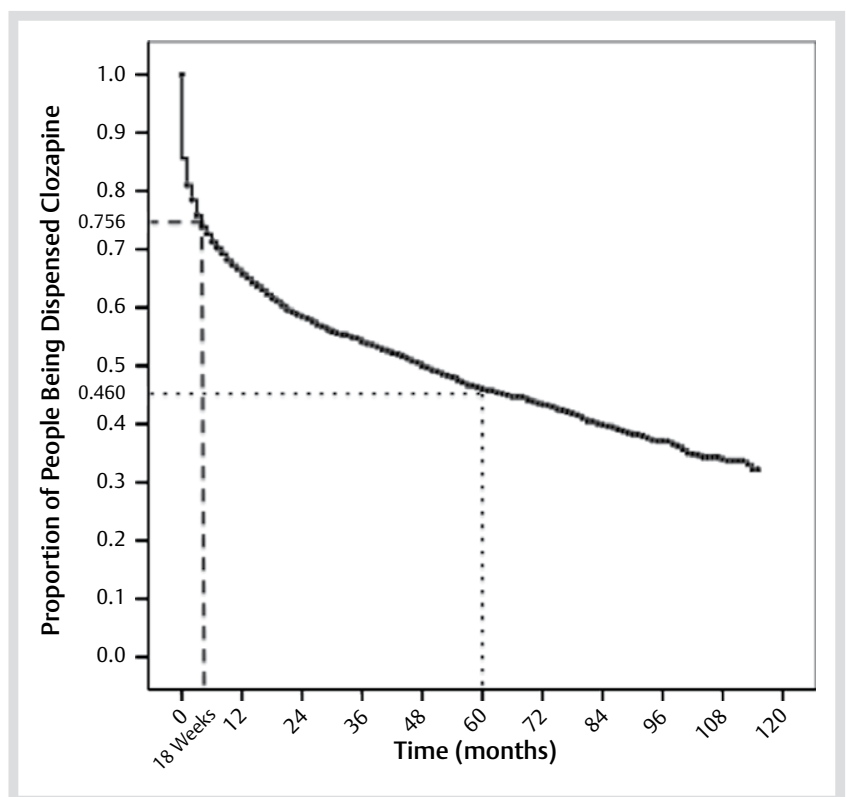

Fig. 3 Actuarial estimate of the proportion of people still dispensed clozapine to cessation of clozapine supply in iPharmacy ${ }^{\circledR}$ dataset.

18.2 to $25.0 \%$ using the higher TRS prevalence estimate of 240 per 100000 .

\section{Mean maintenance dose}

The mean maintenance dose per day for all people who reached clozapine maintenance treatment $(\mathrm{n}=2534 ; 72.7 \%)$ was $364 \mathrm{mg}$ in the first year after starting, $399 \mathrm{mg}$ in the fifth year $(\mathrm{n}=962)$ and $412 \mathrm{mg}$ in the tenth year $(\mathrm{n}=95)$ ( $\odot$ Table 1$)$. The increase in mean dose over the 5 -year $(\mathrm{p}=0.568)$ and 10 -year $(\mathrm{p}=0.452)$ follow-up was not statistically significant.

\section{Duration on clozapine treatment}

At 18 months, the actuarial estimate of the proportion of people still dispensed clozapine from a Queensland public hospital pharmacy was 0.616 (S.E. 0.08) ( $\odot$ Fig. 3). This reduced to 0.460 (S.E. 0.009) at 5 years, and 0.322 (S.E. 0.015 ) at 9.5 years. The median time to cessation was 48 months (95\% Cl 42.45-53.55). More than one in 4 people $(n=950 / 3484 ; 27.3 \%)$ commenced on clozapine did not reach maintenance ( $>18$ weeks treatment). Of 


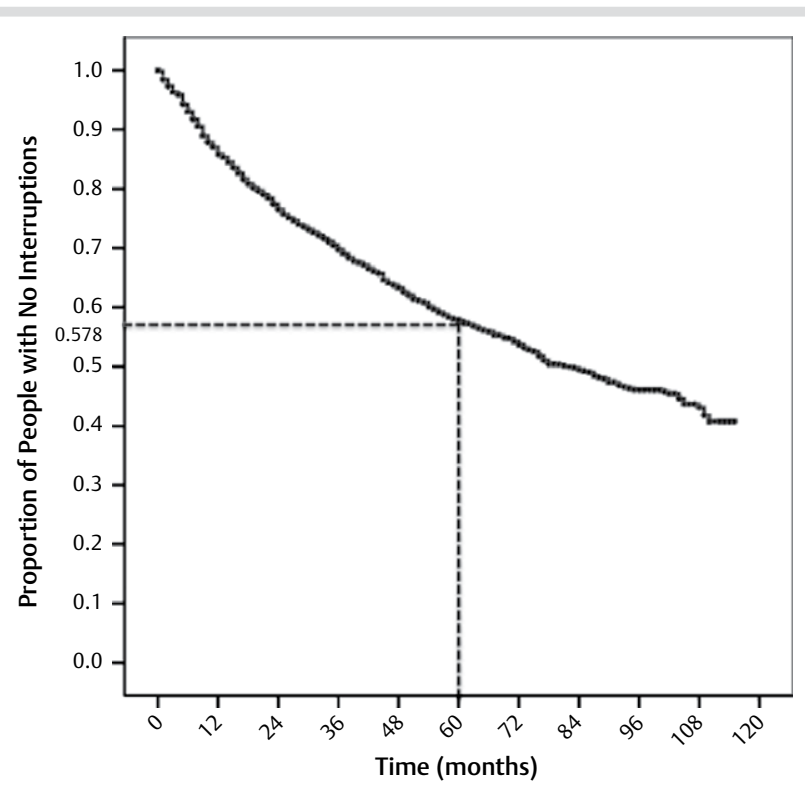

Fig. 4 Actuarial estimate of the proportion of people who had experienced either a short or long interruption to maintenance supply ${ }^{\dagger}$ of clozapine in iPharmacy ${ }^{\circledR}$ dataset. ${ }^{\dagger}$ Maintenance treatment is defined as $>18$ weeks treatment.

these, $52.0 \%(n=494 / 950)$ discontinued within the first 3 weeks. Of those who did reach maintenance treatment, the actuarial estimate of the proportion of people still dispensed clozapine after 5 years was 0.610 (S.E. 0.011 ), with a median time to cessation of 93 months (95\% CI 87-100).

\section{Interruptions to treatment}

Over 5 years of clozapine treatment, the actuarial estimate of the proportion of people who had experienced either a short $(72 \mathrm{~h}$ to 28 days) or long ( $>28$ days) interruption in their clozapine supply was 0.422 (S.E. 0.013) ( $\odot$ Fig. 4). After 9.5 years it was 0.592 (S.E. 0.022). Median time to any interruption was 82 months (95\% CI 72.51-91.49). When we analysed the short and long interruptions separately, the actuarial estimates of the proportion of people who had either a short interruption or a long interruption at 5 years were similar: 0.132 (S.E. 0.014) and 0.120 (S.E. 0.014), respectively. For those people without an interruption in the 2 years post initiation, the rate of any treatment interruption between years 2 to 10 was stable at $4.8 \%$ per year.

\section{Discussion}

$\nabla$

This retrospective study collated a unique dataset containing over $90 \%$ of all people dispensed clozapine in Queensland between 1 January 2004 and 31 December 2013. Despite a 36.4\% increase in the number of people dispensed clozapine from Queensland public hospitals, only one-third of people with TRS were being dispensed clozapine in 2013. This likely reflects an underutilisation of clozapine given clozapine response rates for TRS are 50-60\% [5]. The highest rate of treatment discontinuation was in the first 3 weeks after initiation. For the people who reached maintenance therapy at 18 weeks, over $60 \%$ were still being dispensed clozapine after 5 years.

At 5 years, only 2 in 5 people had an interruption to dispensing. This is noteworthy as people with schizophrenia, similar to other chronic conditions, often have a high rate of treatment interruption due to poor medication adherence [11]. This interruption rate is lower than that reported for treatment of many other chronic physical conditions, such as statins for hypercholesterolaemia [12]. The mean maintenance clozapine dose remained relatively unchanged over the 10 -year period and was similar to other studies [13-16].

Estimates of clozapine use vary greatly worldwide. This is due to variable methods employed to measure use and few studies used dispensing data. In addition, most studies only report use in populations of people with a diagnosis of schizophrenia rather than within the TRS population. A cross-sectional analysis of Danish prescribing data reported total clozapine use increased from 9.0-10.1\% between 1996 and 2007 among people with a diagnosis of schizophrenia in the Danish national registers [13]. A UK study found that clozapine is underused, and there is a 16 -fold variation in prescribing between different postcodes based on spending figures [17]. A US study using Department of Veterans Affairs pharmacy records reported clozapine use increased from 2.1-3\% between 1999 and 2006 among 88,507 veterans with schizophrenia [18]. In New Zealand, a study exploring clozapine use in people with schizophrenia living in the community (using data extracted from prescription records in the clinical file) reported an increase from $21.1-32.8 \%$ between 2000 and 2004 [19]. In Australia, a study in Victoria of 467 outpatients prescribed antipsychotic treatment in 2000 reported that 39\% were on clozapine [14]. Another Australian study used manufacturers' data to estimate the increase in importation of clozapine into Australia from 2000 to 2009, and showed a similar increase in rate of use to our findings [15]. In our study, one in 4 people did not continue treatment past the 18-week stage and of these, almost half had discontinued treatment by 3 weeks. This high rate of early discontinuation may be due to dose-dependent ADRs experienced in the first few weeks of treatment [20]. Some ADRs (myocarditis and neutropenia) necessitate the prescriber to cease clozapine, whilst others (e.g., hypersalivation, sedation, constipation, weight gain, sexual dysfunction) may mean consumers choose to cease clozapine, especially if they are not managed carefully. Clozapine should be slowly uptitrated as per individual response, to minimise dosedependent side effects leading to unnecessary cessation [20]. Alternatively, this high rate of early discontinuation may be due to clozapine not being trialed for an adequate period of time. A response to clozapine can take 6 months or more, particularly when considering functional and symptom improvement [5]. Multidisciplinary team engagement with the consumer and their family or carer(s) and proactive assessment and management of ADRs, especially in the first 18 weeks, alongside monitoring symptoms and functional outcomes over the first 6-12 months, is important to ensure the quality and safe use of clozapine.

We observed that $61.6 \%$ of people were still being dispensed clozapine from Queensland public hospitals at 18 months. This compares favourably to the Clinical Antipsychotic Trials for Intervention Effectiveness (CATIE) study which reported only $36 \%$ of people prescribed olanzapine and $18 \%$ prescribed quetiapine continued treatment at 18 months [21]. In a second stage of CATIE (non-responders randomised to clozapine or another second-generation antipsychotic), the time to discontinuation due to a lack of effect was significantly longer for clozapine than olanzapine, risperidone or quetiapine [22]. The median time to clozapine cessation in the CATIE follow-up was 10.5 months, 
which is considerably shorter than the median of 48 months found in Queensland. In a 15-year naturalistic study of 320 US veterans on clozapine, $57 \%$ had a discontinuation of treatment with a median time of 609 days. This was a broadly similar proportion of interruptions, though with a briefer median duration to interruption, than in our study [23]. A Swedish registry study also found that people on clozapine were more likely to fill their prescriptions than people on other antipsychotics [24].

Our study suggests that clozapine is being underused. It is estimated that $50-60 \%$ of people with TRS would respond to clozapine [5]. Although there will often be barriers to commencing clozapine, such as poor engagement with treating teams and comorbid substance abuse, all people diagnosed with TRS deserve an opportunity to be trialed on clozapine for a sufficient period of time. Underuse of clozapine can have an impact on the individual consumer, their family and society as a whole through a reduction in quality of life, employment and relationships, increased hospitalisation and exposure to sub-optimal antipsychotic treatment such as polypharmacy and high-dose prescribing $[1,25,26]$.

The strength of this study was the development of a large, comprehensive and reliable dataset containing continuous and individual-level clozapine dispensing histories for an entire state over 10 years, derived from routinely collected data. The dataset consisted of 4813 individuals supplied clozapine. Electronic pharmacy records from a closed pharmacy system with the exact strength and quantity of medication supplied, in addition to the frequency of refills, can provide a reasonably accurate record of medication adherence [27]. This study also has limitations. The dataset did not contain any demographic information nor was it directly linked to any hospital admission, medical records or laboratory results to explore ADRs, outcome data, confirm TRS diagnosis, or examine clozapine levels. An additional data linkage exercise was beyond the scope of this project. 2 events during the course of the data collection period created challenges in data analyses. Firstly, from 2010 some people were shifted from Queensland public hospital dispensing to noniPharmacy ${ }^{\circledR}$ dispensing through private hospitals and community pharmacies associated with a hospital. This was seen in the plateau in the number of people dispensed clozapine after 2011 in the iPharmacy ${ }^{\circledR}$ dataset ( $\bullet$ Fig. 1). Further examination revealed the increase in non-iPharmacy ${ }^{\circledR}$ dispensing ( $\bullet$ Fig. 2) corresponded to the plateau in the number of individuals supplied clozapine in the iPharmacy ${ }^{\circledR}$ dataset ( $\bullet$ Fig. 1). This suggested that the number of individuals dispensed clozapine in Queensland continued to increase throughout the study period. It is likely that our estimate of 60 people supplied clozapine per 100000 population in 2013 may be conservative. Secondly, the number of clozapine iPharmacy ${ }^{\circledR}$ dispensings was affected by a statewide contractual change in clozapine manufacturers which subsequently changed the clozapine dose formulations from 25-mg, 50-mg, 100-mg and 200-mg to only 25-mg and 100-mg tablets. The effect of this change can be seen in the slight dip in clozapine items dispensed between 2011 and 2012 (० Fig. 2). The change in dose formulations had no impact on the calculation of the number of individuals dispensed clozapine through iPharmacy ${ }^{\circledR}$ nor on the mean maintenance dose calculations. In conclusion, this study has shown that, despite clozapine being underused in people with TRS, the number of individuals being dispensed clozapine from Queensland public hospitals increased over the study period (adjusted for population growth). Reassuringly, 3 in 4 people initiating clozapine successfully continued beyond the 18-week initiation period, and more than half continued for at least 5 years. Increased use will require a commensurate increase in the monitoring and management of clozapine ADRs. Further evaluation of barriers to initiation and continuation beyond the first month of clozapine is warranted.

\section{Funding}

$\nabla$

This research received no specific grant from any funding agency in the public, commercial, or not-for-profit sectors.

\section{Acknowledgements}

$\nabla$

We thank James Grant for his help with the initial iPharmacy ${ }^{\circledR}$ dataset preparation, David McDougall and Victor Siskind for their assistance in the statistical analysis and Lynette Loy for her insight into clozapine PBS use in Queensland public hospitals.

\section{Declaration of Interest \\ $\nabla$}

TF, DS, KW \& SH have no interests to declare. AW has received research funding, lecturing and/or conference support from Janssen-Cilag, Eli Lilly, AstraZeneca, Roche Diagnostics, Novartis and Douglas Pharmaceuticals, Pfizer, Sanofi-Aventis, Douglas Pharmaceuticals and Wyeth.

\section{Affiliations}

1 Princess Alexandra Hospital, Brisbane, Australia

${ }^{2}$ Metro South Addiction and Mental Health Service, Brisbane, Australia

${ }^{3}$ School of Medicine, The University of Queensland, Brisbane, Australia

${ }^{4}$ School of Pharmacy, The University of Queensland, Brisbane, Australia

${ }^{5}$ Griffith Health Institute, Griffith University, Brisbane, Australia

${ }^{6}$ Faculty of Medical and Health Sciences, University of Auckland, Auckland, New Zealand

\section{References}

1 Farooq S, Taylor M. Clozapine: dangerous orphan or neglected friend? The British Journal of Psychiatry 2011; 198: 247-249

2 Kane J, Honigfeld G, Singer J et al. Clozapine for the treatment-resistant schizophrenic. A double-blind comparison with chlorpromazine. JAMA Psychiatry 1988; 45: 789-796

3 Alessi-Severini S, Biscontri R, Collins $D$ et al. Utilization and costs of antipsychotic agents: a Canadian population-based study, 1996-2006. Psychiatric Services 2008; 59: 547-553

4 Mao P-X, Jiang F, Chen Q et al. Clozapine in China. Pharmacopsychiatry 2008; 41: 1-9

5 Meltzer HY. Treatment of the neuroleptic-nonresponsive schizophrenic patient. Schizophrenia bulletin 1992; 18: 515-542

6 Pharmaceutical Benefits Scheme. Clozapine: Australian Government Department of Human Resources; 2014 [cited 2014 16/10]. Available from http://www.pbs.gov.au/medicine/item/5626D-5627E-5628F-56 29G-5630H-6101D-6102E-6417R-6418T-9632Y

7 Australian Bureau ofStatistics. Australian Demographic Statistics: ABS.2013; [cited 201422/09].Available from http://www.abs.gov.au/AUSSTATS/abs@. nsf/second + level + view? ReadForm \&prodno $=3101.0$ \&viewtitle $=$ Austra lianDemographicStatistics $\sim$ Dec 2013 Latest $~ 19 / 06 / 2014 \& \&$ tabname $=$ Past Future Issues $\&$ prodno $=3101.0$ \&issue $=$ Dec $2013 \&$ num $=$ \&view $=$ \&

8 Saha S, Chant $D$, Welham J et al. A systematic review of the prevalence of schizophrenia. PLoS Med 2005; 2: e141

9 Conley RR, Buchanan RW. Evaluation of treatment-resistant schizophrenia. Schizophrenia bulletin 1997; 23: 663-674

10 Medicare Australia. Pharmaceutical Benefits Schedule Item Reports: Australian Government Department of Human Resources; 2014; [cited 2014 22/09]. Available from http://www.medicareaustralia. gov.au/statistics/pbs_item.shtml 
11 Valenstein M, Blow FC, Copeland LA et al. Poor antipsychotic adherence among patients with schizophrenia: medication and patient factors. Schizophrenia bulletin 2004; 30: 255

12 Benner JS, Glynn RJ, Mogun $H$ et al. Long-term persistence in use of statin therapy in elderly patients. Jama 2002; 288: 455-461

13 Nielsen J, Roge R, Schjerning $O$ et al. Geographical and temporal variations in clozapine prescription for schizophrenia. European Neuropsychopharmacology 2012; 22: 818-824

14 Conley RR, Kelly DL, Lambert TJ et al. Comparison of clozapine use in Maryland and in Victoria, Australia. Psychiatric Services 2005; 56: 320-323

15 Malalagama G, Bastiampillai T, Dhillon R. Clozapine prescription patterns in Australia over the last 10 years. Australian and New Zealand Journal of Psychiatry 2011; 45: 498-499

16 Wheeler AJ, Feetam CL, Harrison J. Pathway to clozapine use: A comparison between a patient cohort from New Zealand and a cohort from the United Kingdom. Clinical drug investigation 2014; 34: 203-211

17 Hayhurst KP, Brown P, Lewis SW. Postcode prescribing for schizophrenia. The British Journal of Psychiatry 2003; 182: 281-283

18 Sernyak MJ, Rosenheck RA. Antipsychotic use in the treatment of outpatients with schizophrenia in the VA from fiscal years 1999 to 2006. Psychiatric Services 2008; 59: 567-569

19 Wheeler A, Humberstone V, Robinson G. Outcomes for schizophrenia patients with clozapine treatment: how good does it get? Journal of Psychopharmacology 2009; 23: 957-965
20 Nielsen J, Damkier P, Lublin H, Taylor D. Optimizing clozapine treatment. Acta Psychiatrica Scandinavica 2011; 123: 411-422 PubMed PMID: 21534935

21 Lieberman JA. Effectiveness of antipsychotic drugs in patients with chronic schizophrenia: efficacy, safety and cost outcomes of CATIE and other trials. Journal of Clinical Psychiatry 2007; 68: e04

22 McEvoy JP, Lieberman JA, Stroup TS et al. Effectiveness of clozapine versus olanzapine, quetiapine, and risperidone in patients with chronic schizophrenia who did not respond to prior atypical antipsychotic treatment. American Journal of Psychiatry 2006; 163: 600-610

23 Davis $M$, Fuller $M$, Strauss $M$ et al. Discontinuation of clozapine: a 15 -year naturalistic retrospective study of 320 patients. Acta psychiatrica Scandinavica 2013

24 Ringback Weitoft G, Berglund M, Lindstrom EA et al. Mortality, attempted suicide, re-hospitalisation and prescription refill for clozapine and other antipsychotics in Sweden - a register-based study. Pharmacoepidemiology and drug safety 2014; 23: 290-298

25 Morgan VA, Waterreus A, Jablensky A et al. People living with psychotic illness in 2010: the second Australian national survey of psychosis. Australian and New Zealand Journal of Psychiatry 2012; 46: 735-752

26 Neil AL, Carr VJ, Mihalopoulos C et al. Costs of psychosis in 2010: Findings from the second Australian National Survey of Psychosis. Australian and New Zealand Journal of Psychiatry 2014; 48: 169-182

27 Osterberg L, Blaschke T. Adherence to medication. New England Journal of Medicine 2005; 353: 487-497 